beneficio disminuyó a medida que aumentó el riesgo inicial de padecer de cáncer de mama. Sin embargo, en estos casos la calidad de la vida estaba asociada con la intensidad de los síntomas. Cuando el riesgo inicial de padecer cáncer de mama era mayor de $35 \%$, la probabilidad de que la HR causara un daño neto era superior al 2,5\%. Específicamente, los beneficios netos fueron de una ganancia de 10,7 meses de vida de buena calidad en mujeres con un bajo riesgo inicial (AVAC = 0,89; IC 95\%: 0,56 a 1,26); de 10,6 meses en mujeres con un riesgo inicial mediano (AVAC $=0,88$; IC 95\%: 0,55 a 1,25); de 8,5 meses en mujeres que tenían un riesgo inicial alto $($ AVAC $=0,71$; IC 95\%: 0,33 a 1,12), y de 1,2 meses enlas que tenían un riesgo inicial muy alto $(\mathrm{AVAC}=$ 0,10; IC $95 \%$ : $-0,78$ a 0,89 ).

La pérdida de AVAC asociada con el empleo de la HR durante cinco años fue de 7 días en mujeres que estaban en perfecto estado de salud y que tenían el menor riesgo inicial $(0,7 \%)$ de sufrir un cáncer de mama, pero podía llegar a 2,5 meses en las que tenían un alto riesgo inicial (12\%).

A pesar de las limitaciones que tiene este trabajo debido a las suposiciones que se hicieron para aplicar los modelos, el análisis de decisión clínica demostró que el uso de la HR para la prevención primaria de enfermedades crónicas en mujeres asintomáticas no está justificado y que los daños netos aumentan a medida que aumenta el riesgo inicial de padecer de cáncer de mama.

En las mujeres que presentan síntomas de menopausia, los beneficios netos asociados con el uso de la HR dependen del impacto de estos síntomas sobre la calidad de la vida. Este impacto varía considerablemente, ya sea por lo variable que puede ser la magnitud de esos síntomas o por la percepción personal de cada mujer acerca del impacto de esos síntomas en su vida cotidiana. Por consiguiente, se debe aplicar un enfoque personalizado para cada mujer, de acuerdo con su riesgo de padecer de cáncer de mama y su percepción del efecto que tienen los síntomas menospáusicos en la calidad de su vida. (Minelli C, Abrams KR, Sutton AJ, Cooper NJ. Benefits and harms associated with hormone replacement therapy: clinical decision analysis. $\mathrm{Br}$ Med J. 2004;328:71.)

\section{Ciertas percepciones y creencias de las mujeres hispanas en Estados Unidos obstaculizan la prevención del cáncer cervicouterino}

La incidencia de cáncer cervicouterino invasor en mujeres de origen hispano en los Estados Unidos de América (EUA) es dos veces mayor que la observada en mujeres blancas que no son hispanas $(15,3$ frente a 7,1 casos $/ 100000$ mujeres, respectivamente). La mortalidad es también superior en las mujeres hispanas (3,4 casos/100 000) que en las mujeres blancas que no son de ascendencia hispana (2,3 casos/100 000) y esta diferencia es aun mayor entre las mujeres hispanas de las comunidades cercanas a la frontera entre Texas, EUA, y México (5,7 frente a 2,9 casos /100 000 mujeres, respectivamente).

El tamizaje anual de las mujeres en edad fértil mediante la prueba de Papanicolaou puede ayudar a prevenir el cáncer cervicouterino invasor gracias a la detección a tiempo de la displasia cervicouterina, estado precanceroso más fácil de tratar. Sin embargo, las mujeres de origen hispano participan menos en los programas de tamizaje de cáncer cervicouterino que las mujeres de otros grupos étnicos. Según la Encuesta de Vigilancia sobre Factores de Riesgo Conductuales, realizada en El Paso, Texas, EUA, 16,6\% de las mujeres hispanas mayores de 18 años nunca se han sometido a la prueba de Papanicolaou. Las razones de esta actitud son los sentimientos de vergüenza, miedo y desesperanza, la falta de conocimientos acerca del cáncer cervicouterino y del tamizaje, y la carencia de un seguro de salud. La mayoría de los estudios que han evaluado los factores que determinan la poca participación de estas mujeres en el tamizaje de cáncer cervicouterino han abarcado a todos los grupos de edad, por lo que no se dispone de suficiente información acerca de los elementos que influyen específicamente en las mujeres jóvenes de este grupo poblacional.

Este estudio correlacionó los antecedentes de participación en el tamizaje de cáncer cervicouterino con las creencias, actitudes y características personales de las mujeres de origen hispano de 18 a 25 años de edad que residen en El Paso, área metropolitana ubicada en la frontera entre México y EUA. Para ello se aplicó una encuesta transversal a una muestra de conveniencia compuesta de 200 mujeres de 18 a 25 años de edad que se consideraban a sí mismas hispanas y que asistían a cursos de superación en varios centros de educación para adultos en El Paso.

Se elaboraron varias escalas basadas en el Modelo de Creencias de Salud (Health Belief Model), desarrollado en la década de 1950 para explicar el poco uso que se hacía de los servicios de tamizaje y prevención establecidos para la detección y el tratamiento tempranos de enfermedades asintomáticas. Las escalas empleadas fueron diseñadas para evaluar la percepción que tenían las mujeres participantes acerca de su predisposición a padecer de cáncer cervicouterino, la gravedad de esta enfermedad, los beneficios del tamizaje mediante la prueba de Papanicolaou y las barreras que les impedían someterse al tamizaje. La encuesta también contaba con una escala de aculturación basada en el Índice de Acultura- 
ción General de Balcazar con respuestas estructuradas mediante una escala de Líkert. Por último, las encuestadas también respondieron preguntas acerca de sus pruebas de Papanicolaou anteriores, los embarazos que habían tenido, su actividad sexual y los métodos anticonceptivos que empleaban, así como el nivel de escolaridad que habían alcanzado y el tipo de seguro médico que tenían.

De las 189 mujeres que respondieron la encuesta, $69 \%$ informaron de haberse realizado alguna vez una prueba de Papanicolaou, pero solo 56\% dijeron habérsela realizado en el último año. Asimismo, $80 \%$ de las encuestadas manifestaron que eran sexualmente activas y de ellas, $63 \%$ dijeron usar algún método anticonceptivo. La mayoría de las encuestadas $(72 \%)$ comprendían la gravedad del cáncer cervicouterino, su susceptibilidad a padecerlo $(89,8 \%)$ y los beneficios que brinda la prueba de Papanicolaou (69,3\%). Sin embargo, solo $61,2 \%$ respondieron que la mayoría de las mujeres jóvenes que conocían se sometían a esta prueba. Tener un mayor nivel de aculturación y considerar que la mayoría de las mujeres jóvenes solteras se someten a la prueba de Papanicolaou se asociaron de manera directa con el hecho de haberse sometido al tamizaje en alguna ocasión.

Algunos elementos percibidos como barreras para hacerse el Papanicolaou mostraron una asociación con el hecho de no haberse realizado nunca esta prueba. Los principales fueron la idea de que la prueba sería dolorosa o causaría vergüenza, que la prueba afectaría a la virginidad, el no saber a dónde dirigirse para hacérsela, el sentimiento de que la pareja no la aprobaría, el criterio de que solo las mujeres que han tenido hijos necesitan la prueba, y la idea de que las mujeres solteras que se hiciesen la prueba serían vistas como mujeres sexualmente activas. Se encontró una asociación más débil con la creencia de que otras mujeres jóvenes se realizaban la prueba.

Los resultados de este estudio demuestran que las mujeres estadounidenses de origen hispano que no tienen una vida sexual activa no siempre perciben la necesidad de hacerse una prueba de tamizaje de cáncer cervicouterino. Las mujeres con un mayor nivel de aculturación presentaron una probabilidad cinco veces más alta de haberse hecho una prueba de Papanicolaou que el grupo de menor nivel de aculturación.

A pesar del diseño transversal de este estudio y de que se basó en datos aportados por las propias mujeres, los resultados revelan que la participación de las mujeres jóvenes de origen hispano en el tamizaje de cáncer cervicouterino es baja en esta comunidad urbana de la frontera entre México y EUA. Además, demostró que determinadas percepciones erróneas obstaculizan el tamizaje en este grupo de mujeres. Estas barreras pueden ser eliminadas mediante intervenciones educativas diseñadas para esta población específica. (Byrd TL, Peterson SK, Chavez R, Heckert A. Cervical cancer screening beliefs among young Hispanic women. Prev Med. 2004;38:192-7.)

\section{Cambios genéticos del vector de la tripanosomiasis pueden tener implicaciones epidemiológicas}

La tripanosomiasis americana o enfermedad de Chagas está reconocida como la enfermedad parasitaria más importante de la Región de las Américas por su impacto social y económico. Esta enfermedad la causa el protozoo flagelado Trypanosoma cruzi y se transmite mediante insectos chupadores de sangre de la subfamilia Triatominae (Hemiptera, Reduviidae). No existen vacunas preventivas, por lo que su control se basa en la eliminación de las poblaciones domésticas del vector.

Para controlar eficazmente la enfermedad de Chagas, es imprescindible conocer la estructura genética de las poblaciones de insectos -incluido el flujo de genes entre las poblaciones domésticas y selváticas-, así como la información acerca de la domesticación y capacidad de diseminación de la infección por parte del vector.

En este trabajo se analizan los cambios cariológicos ocurridos durante la diseminación de Triatoma infestans, el mayor vector de la tripanosomiasis americana, empleando para ello la cuantificación de ADN mediante la citometría de flujo y la caracterización de los patrones de segmentos heterocromáticos o bandas $\mathrm{C}$ de los cromosomas.

Se encontraron dos grupos cromosómicos de T. infestans: uno en las muestras del área andina de Bolivia y Perú y otro en las muestras procedentes de zonas bajas (Argentina, Paraguay, Brasil, Uruguay y el Chaco boliviano). Estos grupos parecen estar bien delimitados y restringidos a áreas geográficas específicas, ya que no se encontraron formas intermedias. Ambos grupos presentaron características diferentes: las muestras andinas presentaron de 14 a 20 autosomas de bandas $C$, mientras que las de otras partes tenían solo de 4 a 7 autosomas; las muestras andinas presentaron un bloque heterocromático en el cromosoma $X$ que estaba ausente en las muestras de otros lugares; y las muestras andinas contenían aproximadamente $30 \%$ más de $\mathrm{ADN}$ por célula que las demás.

Estos resultados y la información histórica y genética disponible hasta el momento indican que T. infestans era originalmente una especie selvática que poblaba la región andina de Bolivia y que tenía una gran cantidad de ADN y de heterocromatina. La 